\title{
The Hymenoptera Genome Database
}

Mónica C. Muñoz-Torres, Justin T. Reese, Christopher P. Childers, Anna K. Bennett, Jaideep P. Sundaram, Donald C. Vile and Christine G. Elsik.

GEORGETOWN UNIVERSITY, DEPARTMENT OF BIOLOGY, WASHINGTON, DC 20057. U.S.A.

\section{HGD IN A NUTSHELL}

The HYMENOPTERA GENOME DATABASE (HGD) is a relational database that implements open-source software and components, providing access to curated data CONTRIBUTED BY an extensive, active research COMMUNITY. HGD contains up-to-date versions of the GENOME ANNOTATIONS for honey bee and its pathogens (http://BeeBase.org), jewel wasps (http://NasoniaBase.org), and a portal to the genomes of six species of ants. Together, these species cover approximately 200 MY in the phylogeny of Hymenoptera, allowing to

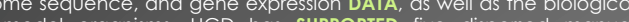
knowledge of relaied model organisms. HGD has SUPFOKTED five dispersed manuc the

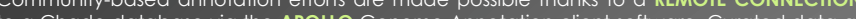
o a Chado dald HGD incluales predicted and annotated gene sets supported with evidence tracks such as

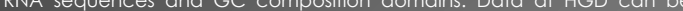
QUERIED using genome browsers and / or BLAST/PSI-BLAST servers, and it may also be downloaded to perform LOCAL SEARCHES. We encourage the public to access and contribute data to HGD a:

$h+t p: / / H$ y m e nop teraGe $n \circ m e$.

DATA EXCHANGE IN THE HYMENOPTERA GENOME DATABASE
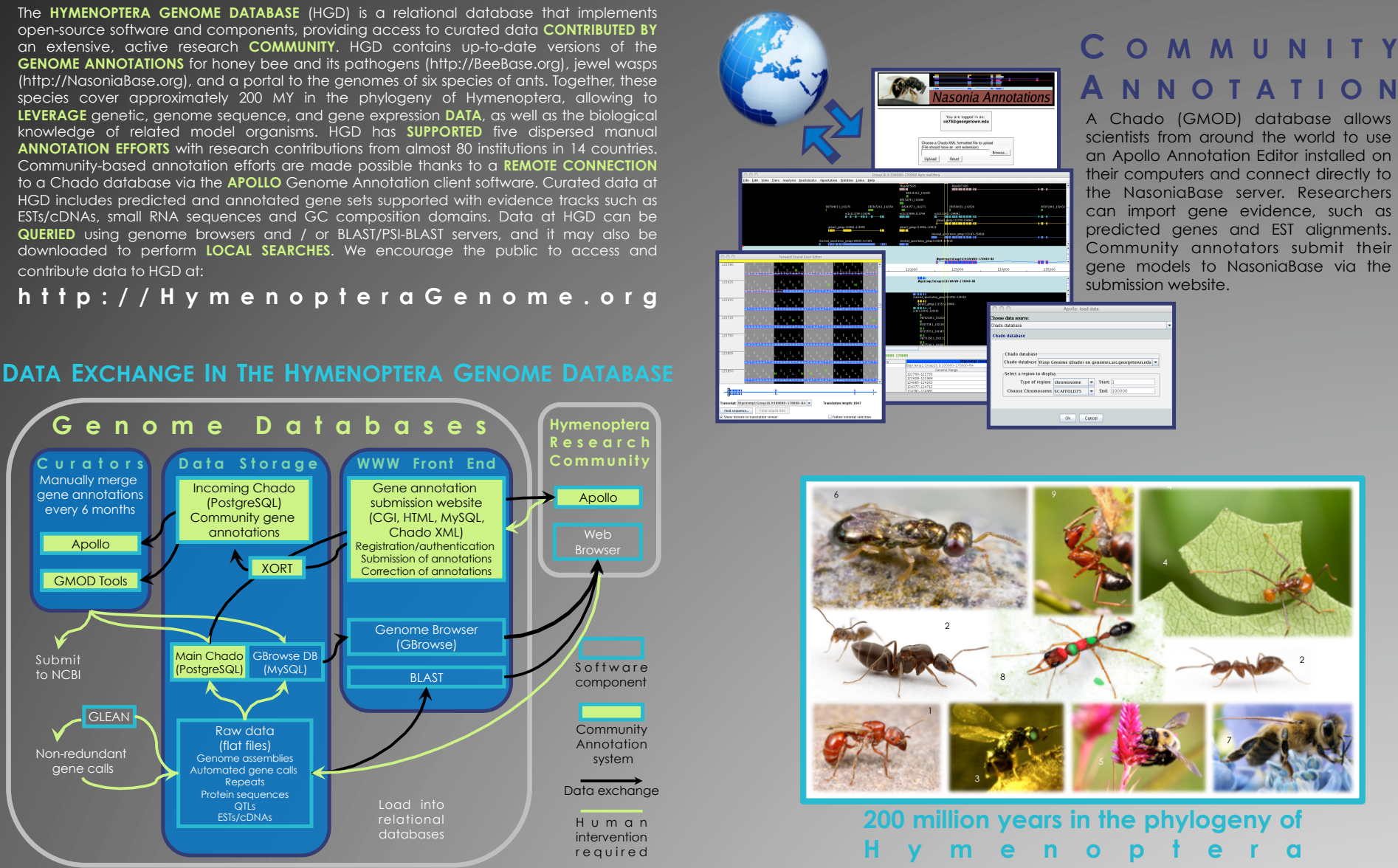

United States Department of Agriculture
National Institute of Food and Agriculture
GEORGETOWNCUNIVERSTI

\section{USDA}

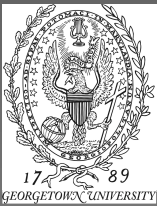

O M M U N I T Y Apollo Annotation Editor installed on EST alignments. Community annotators submit their

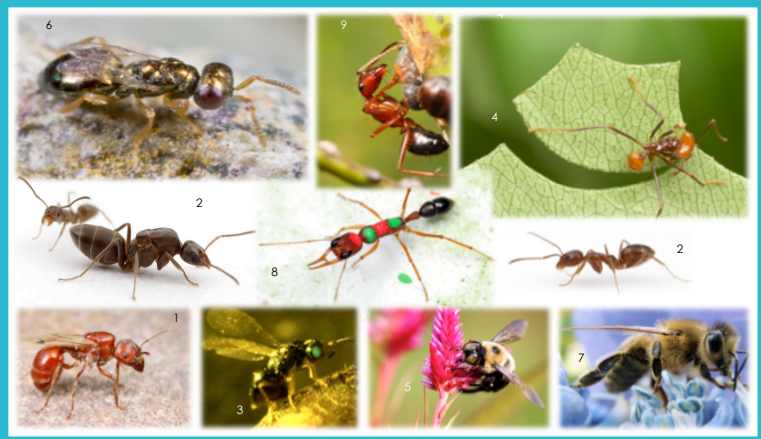

200 million years in the phylogeny of

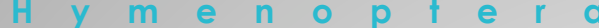

\section{A VAILABLE RESOURCES}

GBrowse \& WIKI PAGES

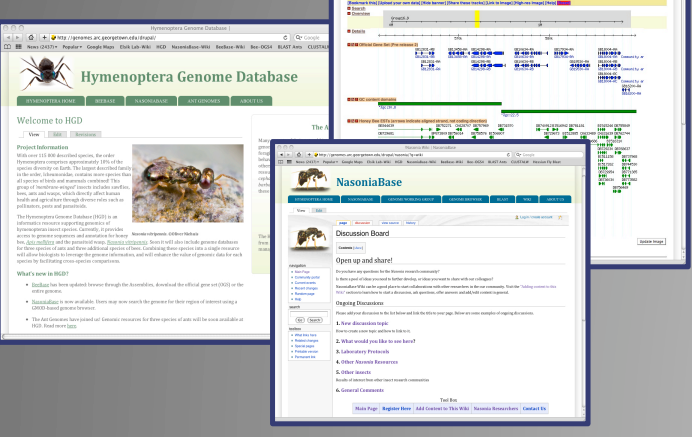

The Hymenoptera Genome Database includes BeeBase, NasoniaBase and the Ant Genomes Portal. BeeBase resources currently include honey bee GBrowse for assemblies and superscaffolds, BLAST, PSI-BLAST, a CMAP comparative map viewer, honey bee pathogen GBrowse, and a sequence query and download webpage. NasoniaBase includes a genome browser, an Apollo annotation database and an annotation community submission website. Future plans include QTL viewer. SNP/Haplotype block GBrowse interface, gene expression annotation query with cross-species comparison functions, and Apis-Nasonia Synteny Broswer. In addition, genes will be annotated with GO terms, and new GO terms will be developed for Hymenoptera species. Gene pages containing annotations, database cross-references, links to other insect MODs, NCBI, Entrez Gene, NCBI Homologene, OrthoMCL, Uniprot, and internal connections to genome viewer, gene expression, SNP and phenotype information will be developed.

FunDING: NRI 2008-35302-18804 from the USDA National Institute of Food and Agriculture. 